\title{
BMJ Open Acceptability of bisphosphonates among patients, clinicians and managers: a systematic review and framework synthesis
}

\author{
Zoe Paskins, ${ }^{1,2}$ Fay Crawford-Manning (D) , ${ }^{1,2}$ Elizabeth Cottrell (D) , ${ }^{1}$ Nadia Corp, ${ }^{1}$ \\ Jenny Wright, ${ }^{1}$ Clare Jinks, ${ }^{1}$ Simon Bishop, ${ }^{3}$ Alison Doyle, ${ }^{4}$ Terence Ong, ${ }^{5}$ \\ Neil Gittoes, ${ }^{6}$ Jo Leonardi-Bee, ${ }^{7}$ Tessa Langley, ${ }^{8}$ Robert Horne, ${ }^{9}$ Opinder Sahota ${ }^{10}$
}

To cite: Paskins Z, CrawfordManning F, Cottrell E, et al. Acceptability of bisphosphonates among patients, clinicians and managers: a systematic review and framework synthesis. BMJ Open 2020;10:e040634. doi:10.1136/ bmjopen-2020-040634

- Prepublication history and additional material for this paper are available online. To view these files, please visit the journal online (http://dx.doi. org/10.1136/bmjopen-2020 040634).

Received 18 May 2020 Revised 01 September 2020 Accepted 13 0ctober 2020

Check for updates

(C) Author(s) (or their employer(s)) 2020. Re-use permitted under CC BY. Published by BMJ.

For numbered affiliations see end of article.

Correspondence to

Dr Zoe Paskins;

z.paskins@keele.ac.uk

\section{ABSTRACT}

Objective To explore the acceptability of different bisphosphonate regimens for the treatment of osteoporosis among patients, clinicians and managers, payers and academics.

Design A systematic review of primary qualitative studies. Seven databases were searched from inception to July 2019. Screening, data extraction and quality assessment of full-articles selected for inclusion were performed independently by two authors. A framework synthesis was applied to extracted data based on the theoretical framework of acceptability (TFA). The TFA includes seven domains relating to sense-making, emotions, opportunity costs, burden, perceived effectiveness, ethicality and selfefficacy. Confidence in synthesis findings was assessed. Setting Any developed country healthcare setting. Participants Patients, healthcare professionals, managers, payers and academics.

Intervention Experiences and views of oral and intravenous bisphosphonates.

Results Twenty-five studies were included, mostly describing perceptions of oral bisphosphonates. We identified, with high confidence, how patients and healthcare professionals make sense (coherence) of bisphosphonates by balancing perceptions of need against concerns, how uncertainty prevails about bisphosphonate perceived effectiveness and a number of individual and service factors that have potential to increase self-efficacy in recommending and adhering to bisphosphonates. We identified, with moderate confidence, that bisphosphonate taking induces concern, but has the potential to engender reassurance, and that both side effects and special instructions for taking oral bisphosphonates can result in treatment burden. Finally, we identified with low confidence that multimorbidity plays a role in people's perception of bisphosphonate acceptability.

Conclusion By using the lens of acceptability, our findings demonstrate with high confidence that a theoretically informed, whole-system approach is necessary to both understand and improve adherence. Clinicians and patients need supporting to understand the need for bisphosphonates, and clinicians need to clarify to patients what constitutes bisphosphonate treatment success. Further research is needed to explore perspectives of male patients and those with multimorbidity receiving
Strengths and limitations of this study

- Comprehensive search strategy.

- Robust framework synthesis underpinned by theory.

- Inclusion of clinician and manager views in addition to patient perspectives.

- Use of Grades of Recommendation, Assessment, Development, and Evaluation Confidence in the Evidence from Qualitative Reviews to give confidence in findings

- Qualitative studies reviewed for inclusion were frequently not specific about the anti-osteoporosis drugs participants were taking, meaning we may have missed papers or over-interpreted findings.

bisphosphonates, and patients receiving intravenous treatment.

PROSPERO registration number CRD42019143526.

\section{INTRODUCTION/BACKGROUND}

Osteoporosis is a disease that is characterised by skeletal fragility and changes in bone microarchitecture resulting in increased risk of fractures with no or low trauma. ${ }^{1}$ The management and care of people with low trauma or fragility fractures results in considerable societal economic burden, annual cost in the UK alone is $£ 4.4$ billion. ${ }^{2}$ Furthermore, the personal impact of fragility fractures is considerable, with potential deleterious effects on physical and psychological health, ability to live independently and increased risk of death. Many of these fractures are potentially preventable with appropriate cost effective and clinically effective drug treatments such as bisphosphonates, the mainstay of osteoporosis treatment. However, the success of treatment depends on patients initiating (starting), executing (or implementing-taking correctly) and persisting (continuing) medication; collectively these 
processes are described as adherence. Adherence with osteoporosis medications is notoriously poor and reported to be poorer than other disease areas. Oral bisphosphonate persistence rates at 1 year are commonly estimated between $16 \%$ and $60 \%{ }^{3}$ Worldwide, many people who would benefit from osteoporosis drugs are not receiving them, and this treatment gap has been described as an 'osteoporosis crisis'. The treatment gap is compounded by poor adherence which results in potentially preventable fragility fractures with their associated burden for patients and their carers, difficulties in professionalpatient relationships, and wasted healthcare resources. ${ }^{5}$

There are a number of different bisphosphonates, some are administered orally, others intravenously. A variety of regimes in terms of dose frequency also exists. Alendronic acid, an oral once-weekly bisphosphonate, is considered first-line and most commonly used. ${ }^{6}$ Bisphosphonates work to reduce fracture risk. A recent network meta-analysis demonstrated that bisphosphonate treatment reduces the risk of fragility fracture (depending on site) by $33 \%-54 \% .^{7}$ Oesophageal or gastrointestinal related side effects are the most common adverse effects of oral bisphosphonate use. To counter these, patients taking oral bisphosphonates are required to remain upright and fast for half an hour after ingestion. Rare side effects of bisphosphonates include osteonecrosis of the jaw and atypical femur fractures, both of which have received significant media attention. Such media reports are temporally related to declining bisphosphonate use. ${ }^{7}$ Due to the gastrointestinal side effects and special instructions for taking oral treatment, it has been suggested that alternative bisphosphonate regimens, for example, annual intravenous zoledronic acid, may promote longterm adherence. ${ }^{8-11}$ Studies to date which have examined patient preferences for osteoporosis treatment, suggest that patients prefer injections given less frequently ${ }^{12-14}$; however, research in other chronic diseases shows that although adherence is improved with less frequent medications, that patients prefer oral to injection treatment. ${ }^{15}$ In osteoporosis, the majority of studies that explore patient preferences employ quantitative methods, for example, discrete choice experiments, where patients are asked to choose between hypothetical treatments in regards to various attributes (eg, efficacy, side effects, route and frequency of administration). ${ }^{13}$ Such studies cannot provide comprehensive insight into patient views, experiences or the explanations for these preferences.

In order to fully understand the osteoporosis treatment gap, and ultimately improve adherence, it is important to understand perspectives of all relevant stakeholders: patients, healthcare professionals (HCPs), managers, payors and academics. ${ }^{16} 17$ This can be achieved using the lens of 'acceptability', defined as 'a multi-faceted construct that reflects the extent to which people delivering, or, receiving a healthcare intervention consider it to be appropriate, based on anticipated or experienced cognitive and emotional responses to the intervention'. ${ }^{18}$ In the context of a research programme designed to determine the research agenda for optimising bisphosphonate treatment, the primary aim of this systematic review is to explore the acceptability of different bisphosphonates regimens among patients, and clinicians and managers.

\section{METHODS}

We conducted a systematic review and framework synthesis of qualitative studies exploring patient and clinician views and experiences of bisphosphonates. The conduct and reporting of this review followed the Preferred Reporting Items for Systematic Reviews and Meta-Analyses (PRISMA) guidelines (see online supplemental file 1 for PRISMA checklist).

\section{Eligibility}

To be eligible for inclusion, studies needed to report on patients', clinicians', academics' and/or manager/ payers' experiences and preferences regarding bisphosphonate regimes for adults ( $\geq 18$ years) with osteoporosis. Bisphosphonates needed to be mentioned by name, or there needed to be sufficient information that was specific to bisphosphonate (eg, reference to the special instructions for use of oral bisphosphonates), to deduce that study findings related to bisphosphonates, as agreed by two clinically experienced authors independently. Papers describing experiences of osteoporosis more generally were included if there were findings relating to bisphosphonate treatment in the study abstract. Studies were only included if they were qualitative in design, or mixed methods with a qualitative component, relevant to a developed country setting and written in English language. Studies were excluded that involved paediatric patients; patients and clinicians receiving/recommending other treatments for osteoporosis; and studies in which bisphosphonates were being used for other indications (eg, malignancy or Paget's disease).

\section{Search methods}

Systematic searches were conducted in seven bibliographic databases (MEDLINE, EMBASE, AMED, CINAHLPlus, PsycINFO, ASSIA, and Web of Science (Social Science Citation Index and Conference Proceedings Citation Index-Social Science and Humanities)) from inception to 15 July 2019. The search strategy used database subject headings and text word searching in title, abstract or keywords, combining terms for: (1) bisphosphonates; (2) experiences and preferences; and (3) qualitative research, based on DeJean $e t a l$ s search filter (see online supplemental file 2 for full MEDLINE search strategy). ${ }^{19}$ Search terms were adapted as appropriate for each database platform.

In addition, grey literature was searched (DART Europe, Open Grey and National Digital Library of Theses and Dissertations); the reference lists of all included studies and relevant systematic reviews identified were checked and key studies were citation tracked. 


\section{Study selection}

Two-stage screening of articles against eligibility criteria was undertaken. First, titles and abstracts were screened, then full texts. At both stages screening was conducted by sets of two reviewers independently (NC, EC, ZP) and articles were excluded by agreement. Disagreements were resolved through discussion or by third reviewer adjudication.

\section{Data extraction}

For each paper data extraction was completed independently by two researchers (ZP and JW or EC and FM). Key findings from the results sections of papers relating to bisphosphonates were extracted; a 'key finding' was defined as any sentence or statement relating to views or experiences of bisphosphonates from the results section of the paper or abstract. Wherever possible, the key finding was extracted as written by the author, with minimal edits only for clarification, description of context or for consistency across papers. For each paper, two authors extracted key findings independently, and subsequently agreed a final list of key findings for each paper. Data were also extracted on participant numbers and demographics, data collection technique, setting and country. Additionally, if available for patients, information was extracted on their bisphosphonate use including type of drug and current status (adherent, non-adherent, decliner).

\section{Quality appraisal}

The quality of each study was assessed using the Critical Appraisal Skills Programme (CASP) qualitative tool. This tool consists of 10 items split into 3 sections (qualitative suitability, data analysis and overall quality) (online supplemental file 2). The first two sections consist of items related to qualitative suitability and data analysis, which were evaluated as 'yes', 'no', 'unclear' or 'partial'. The final question was an assessment based on the overall quality of the paper; this was informed by response to the previous items (indicating methodological quality) and by the relevance of the study to the review objectives and was rated as 'high', 'moderate' or 'low'. All papers were quality appraised by two researchers independently (FM, $\mathrm{SB}, \mathrm{JW}$ ). Disagreements were resolved through discussion with a fourth reviewer $(\mathrm{ZP})$.

\section{Synthesis}

We used a framework synthesis approach informed by the 'best fit' model described by Carroll et al. ${ }^{20}$ The 'best fit' method offered a means to test, reinforce and build on an existing published model, conceived for a different but relevant purpose. This approach was chosen as a published theory was identified from the literature that conceptualised acceptability-the theoretical framework of acceptability (TFA) ${ }^{18}$ The TFA is a relatively new framework which was developed to inform the understanding of acceptability of complex interventions, and consists of seven constructs: affective attitudes-the emotions elicited by an intervention; intervention coherence-the extent to which an intervention makes sense; perceived effectiveness-the perceived extent to which intervention will achieve purpose; burden-the amount of effort required to participate in an intervention; selfefficacy-individual's confidence that they can perform the behaviour(s) required to participate in the intervention; opportunity-costs-the extent to which benefits, profits, or values must be given up to engage in an intervention; and ethicality - the extent to which an intervention has a good fit with an individual's values. The framework also incorporates temporal perspectives on anticipated and experienced acceptability at three time points before (prospective), during (experienced) and after (retrospective) experience of an intervention.

The TFA has not previously been used to evaluate drug acceptability. We anticipated the seven constructs of the TFA would be relevant to engagement with drug treatment; for example, burden could relate to treatment burden associated with administrating the drug or side effects. However, one aspect which did not appear to be explicitly conceptualised within the framework was patient beliefs about medicines. Studies across a range of long term conditions, healthcare systems and cultures have consistently shown that engagement with treatment is influenced by patients' personal evaluation of the medicine in question. ${ }^{21}$ Particularly important is how they judge their personal need for treatment relative to their concerns about it. For this reason, we therefore included the Necessity Concerns Framework (NCF), ${ }^{21}$ to further explore the TFA domain relating to intervention coherence.

The first author initially conducted inductive open coding on the data extracted, before mapping the codes to a draft framework derived from a priori themes (the domains of the TFA). Authors then met to first discuss the themes and compare findings for each study and the 'fit' to the draft framework. A preliminary synthesis was achieved using tabulation of studies, organising the studies into groups relating to temporal perspectives and research question, and exploring relationships between studies and between groups.

A final coding framework was agreed at a second meeting of authors. A second author (FM) recoded the original key findings, where necessary, to the new framework to ensure all findings were represented. Finally, relationships between themes and TFA and NCF domains were explored by further group discussion. We used the Grades of Recommendation, Assessment, Development, and Evaluation Confidence in the Evidence from Qualitative Reviews (GRADE-CERQual) approach to determine confidence in our synthesised findings. ${ }^{22}$

\section{Patient and public involvement}

Members of the Nottingham National, Royal Osteoporosis Society Support Group were involved in a series of meetings to discuss the design of the overarching research programme in which this study sits, and confirmed that 


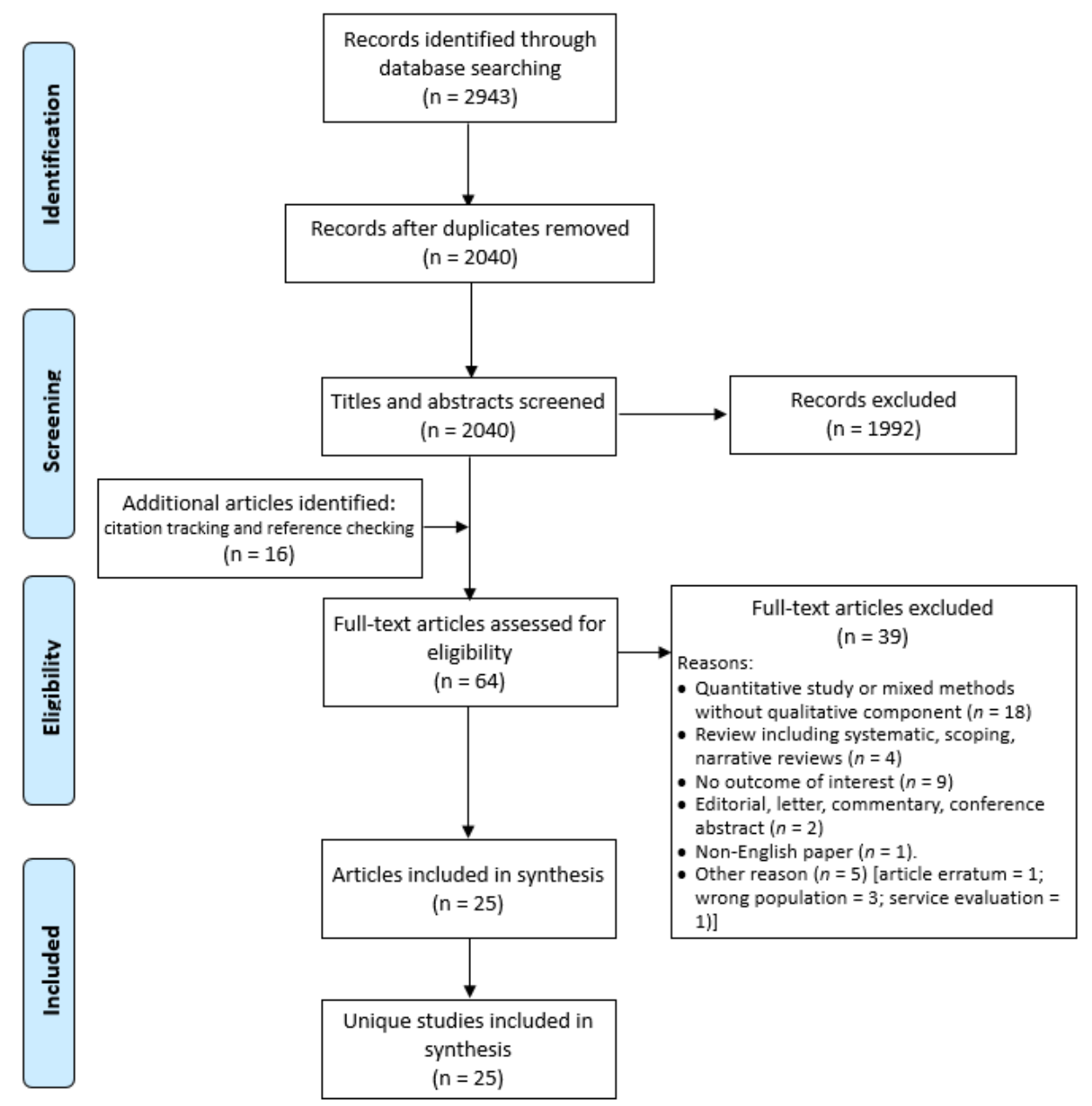

Figure 1 Preferred Reporting Items for Systematic Reviews and Meta-Analyses diagram.

understanding acceptability of bisphosphonates from a range of perspectives was important. Patient were not directly involved in the conduct of this study.

\section{RESULTS}

The literature search identified 2040 unique articles, of which 25 met eligibility criteria (figure 1), a summary of the studies is shown in table 1 .

The included studies were categorised into three groups: perceptions of osteoporosis generally ${ }^{23-29}$; healthcare service delivery issues unrelated to osteoporosis (de-prescribing ${ }^{30}$ and inter-professional communication in primary care ${ }^{31}$ ) and studies specific to osteoporosis treatments. The latter group was further subdivided into: those examining treatment barriers ${ }^{1632-36}$; adherence $^{37-39}$; decision-making $^{40-44}$; or bisphosphonate-related side effects. ${ }^{45} 46$ Only one study examining adherence and one examining decision making had research questions which specifically related to bisphosphonates. ${ }^{38} 43$

The majority (23) of studies were conducted in North America or Europe. Eighteen studies explored patient views, ${ }^{1623-27333537-46}$ of which eight included men, and one study recruited patients taking anti-osteoporosis drugs for glucocorticoid-induced osteoporosis. ${ }^{36}$ Twelve studies explored HCPs' views, ${ }^{16}$ 28-32 34-36 394243 and two studies interviewed managers. ${ }^{16} 34$ No studies included academic or payor participants. Of the 18 studies that included patients, 10 studies described how many of the patients were on anti-osteoporotic medication, however, only two reported the specific type of medication. Only one study reporting patient experience of receiving intravenous bisphosphonate. ${ }^{27}$

The findings related to quality appraisal are summarised in table 2. The most common limitations of the included studies were lack of description of author reflexivity, lack of depth of analysis, use of normative statements and relatively small samples or studies conducted in a single site which may limit transferability of the findings. Furthermore, although the characteristics of the sample were generally reasonably described, in order to address our research question, we required information about medication use of participants which was frequently not described.

Using the CASP tool, $12(48 \%)$ studies were scored as high value and the remaining 13 (52\%) studies as moderate value. For $5 / 13(38 \%)$ studies scored as 


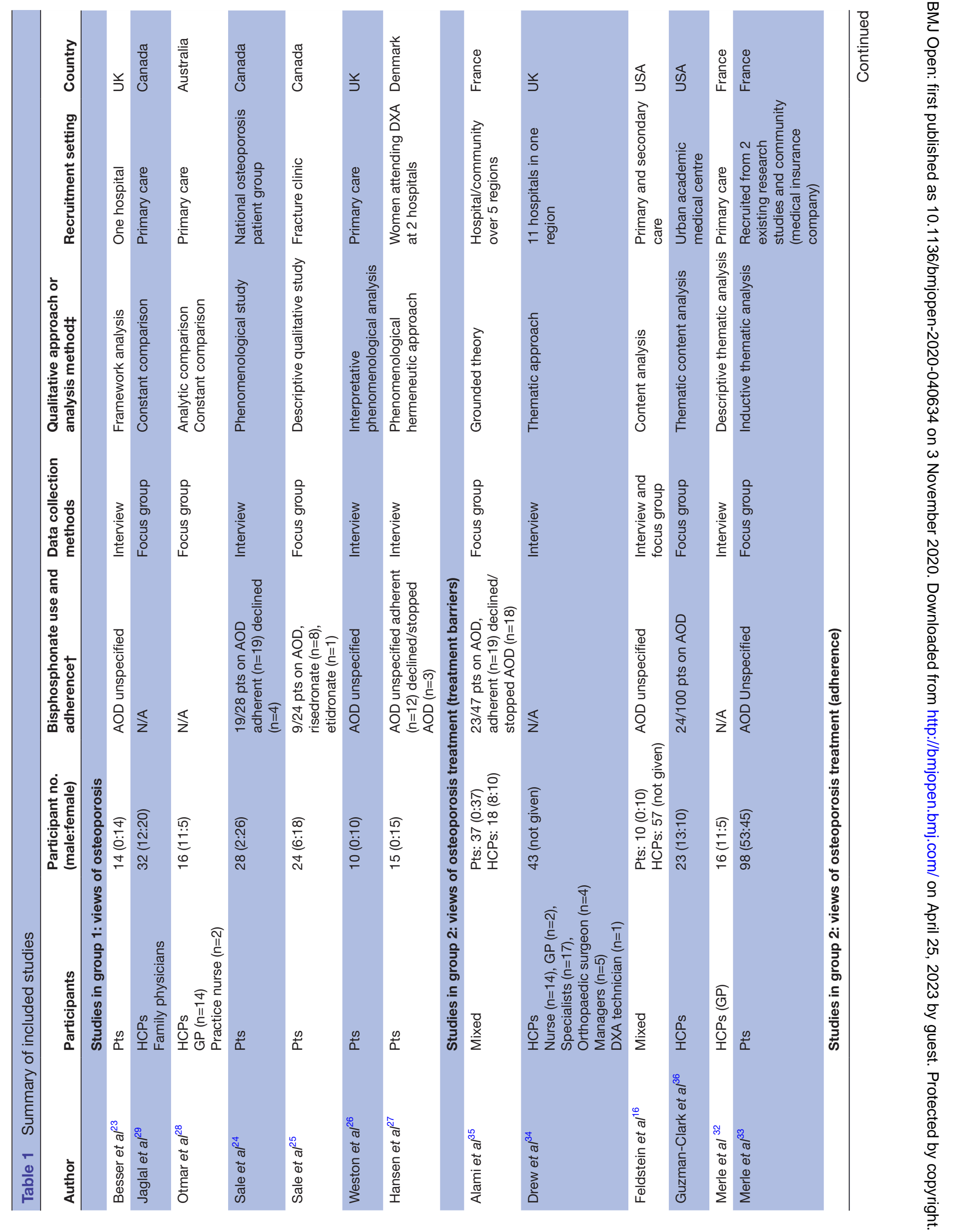




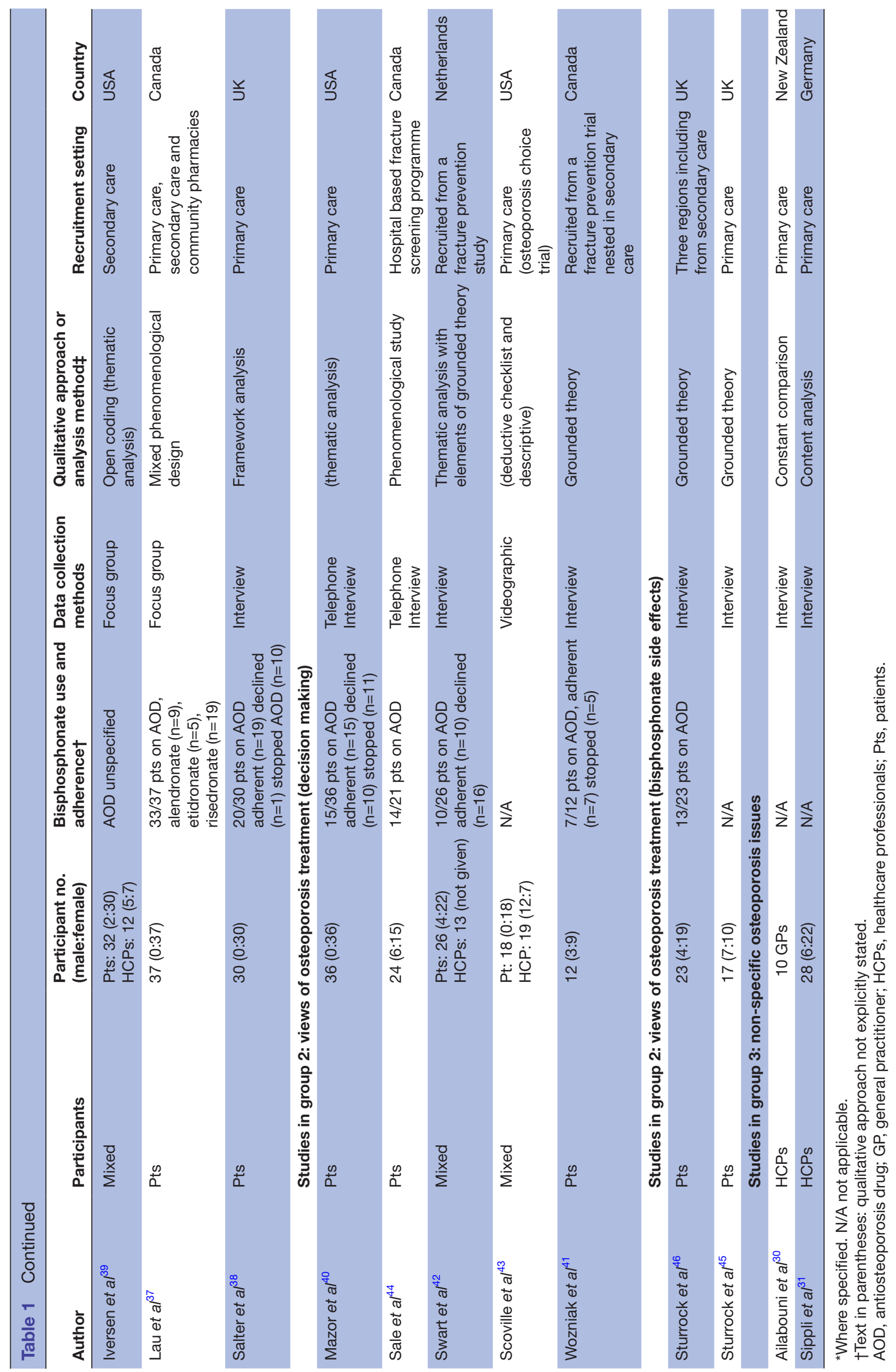

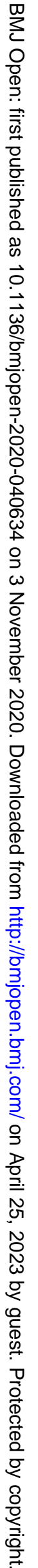


Table 2 Quality appraisal

\section{CASP tool question}

\begin{tabular}{|c|c|c|c|c|c|c|c|c|c|c|c|}
\hline Author & 1 & 2 & 3 & 4 & 5 & 6 & 7 & 8 & 9 & 10 & Comments† \\
\hline \multicolumn{12}{|c|}{ Group 1: views of osteoporosis } \\
\hline Besser et $a^{23}$ & $\checkmark$ & $\checkmark$ & $\checkmark$ & $\mathrm{p}$ & $\checkmark$ & & $\checkmark$ & $\mathrm{p}$ & $\checkmark$ & Moderate & $\begin{array}{l}\text { Small sample, no mention of data saturation, limited to } \\
\text { 'psychological' factors affecting adherence (discounting otr } \\
\text { factors by omission) and some use of normative statements }\end{array}$ \\
\hline Jaglal et $\mathrm{al}^{29}$ & $\checkmark$ & $\checkmark$ & $\checkmark$ & $\checkmark$ & $\checkmark$ & u & $\checkmark$ & $\checkmark$ & $\checkmark$ & Moderate & Few findings relevant to our research question \\
\hline Otmar et $a l^{28}$ & $\checkmark$ & $\checkmark$ & $\checkmark$ & $\checkmark$ & $\checkmark$ & & $\checkmark$ & $\checkmark$ & $\checkmark$ & Moderate & $\begin{array}{l}\text { Well conducted study, but limited findings relating to } \\
\text { bisphosphonates }\end{array}$ \\
\hline Sale et $\left.a\right|^{24}$ & $\checkmark$ & $\checkmark$ & $\checkmark$ & $\checkmark$ & $\checkmark$ & u & $\checkmark$ & $\checkmark$ & $\checkmark$ & High & \\
\hline Sale et $a l^{25}$ & $\checkmark$ & $\checkmark$ & $\checkmark$ & $p$ & $\checkmark$ & u & $\checkmark$ & $p$ & $\checkmark$ & Moderate & $\begin{array}{l}\text { Small single site study, although data saturation reached. } \\
\text { Language does not always appear to match approach (eg, } \\
\text { reporting patient 'inability' to link fractures to osteoporosis } \\
\text { suggests prior normative assumptions) }\end{array}$ \\
\hline
\end{tabular}

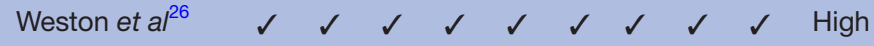

Group 2: views of osteoporosis treatment

\begin{tabular}{|c|c|c|c|c|c|c|c|c|c|c|c|}
\hline Alami et $a /^{35}$ & $\checkmark$ & $\checkmark$ & $\checkmark$ & $\checkmark$ & $\checkmark$ & & $\checkmark$ & $\checkmark$ & $\checkmark$ & High & \\
\hline Drew et $\left.a\right|^{34}$ & $\checkmark$ & $\checkmark$ & $\checkmark$ & $\checkmark$ & $\checkmark$ & u & $\checkmark$ & $\checkmark$ & $\checkmark$ & High & \\
\hline Feldstein et $a l^{16}$ ) & $\checkmark$ & $\checkmark$ & $\checkmark$ & $\checkmark$ & $\checkmark$ & u & $\checkmark$ & $\checkmark$ & $\checkmark$ & High & \\
\hline Merle et $a^{32}$ & $\checkmark$ & $\checkmark$ & $\checkmark$ & $p$ & $\checkmark$ & u & $\checkmark$ & u & $\checkmark$ & Moderate & $\begin{array}{l}\text { Small sample (although data saturation reached) without } \\
\text { attempt to structure to population and analysis lacks depth to } \\
\text { answer our objective relating to bisphosphonate acceptability }\end{array}$ \\
\hline Merle et $a /^{33}$ & $\checkmark$ & $\checkmark$ & $\checkmark$ & $\checkmark$ & $\checkmark$ & $\mathrm{u}$ & $\checkmark$ & $\checkmark$ & $\checkmark$ & Moderate & $\begin{array}{l}\text { Limited information relevant to our research question in view of } \\
\text { general focus on osteoporosis }\end{array}$ \\
\hline Iversen et $\left.a\right|^{39}$ & $\checkmark$ & $\checkmark$ & $\checkmark$ & $p$ & $\checkmark$ & & $\checkmark$ & $p$ & $\checkmark$ & Moderate & $\begin{array}{l}\text { Single centre study, although data saturation reached, limited } \\
\text { information on coding/analysis and no discussion of findings } \\
\text { with relevance to wider literature }\end{array}$ \\
\hline Lau et $a l^{37}$ & $\checkmark$ & $\checkmark$ & $\checkmark$ & $\checkmark$ & $\checkmark$ & & $\checkmark$ & $\checkmark$ & $\checkmark$ & High & \\
\hline Sale et $a l^{44}$ & $\checkmark$ & $\checkmark$ & $\checkmark$ & $\checkmark$ & $\checkmark$ & u & $\checkmark$ & $\checkmark$ & $\checkmark$ & High & \\
\hline Swart et $a l^{42}$ & $\checkmark$ & $\checkmark$ & $\checkmark$ & $\checkmark$ & $\checkmark$ & $\checkmark$ & $\checkmark$ & $\checkmark$ & $\checkmark$ & High & \\
\hline Scoville et $a^{43}$ & $\checkmark$ & $\checkmark$ & $\checkmark$ & $\checkmark$ & $\checkmark$ & u & $\checkmark$ & $\checkmark$ & $\checkmark$ & Moderate & $\begin{array}{l}\text { Well conducted videographic study, but data coded against } \\
\text { deductive categories of reasons to reject treatment, so limited } \\
\text { potential to inform our objective about acceptability }\end{array}$ \\
\hline Wozniak et al ${ }^{41}$ & $\checkmark$ & $\checkmark$ & $\checkmark$ & $\checkmark$ & $\checkmark$ & u & $\checkmark$ & $\checkmark$ & $\checkmark$ & High & \\
\hline Sturrock et al ${ }^{46}$ & $\checkmark$ & $\checkmark$ & $\checkmark$ & $\checkmark$ & $\checkmark$ & u & $\checkmark$ & $\checkmark$ & $\checkmark$ & High & \\
\hline Sturrock et $a l^{45}$ & $\checkmark$ & $\checkmark$ & $\checkmark$ & $\checkmark$ & $\checkmark$ & & $\checkmark$ & $\checkmark$ & $\checkmark$ & Moderate & Aim only partially relevant to study question \\
\hline
\end{tabular}

Continued 
Table 2 Continued

CASP tool question*

$\begin{array}{lllllllllllll}\text { Author } & 1 & 2 & 3 & 4 & 5 & 6 & 7 & 8 & 9 & 10 & \text { Comments }\end{array}$

${ }^{*}$ Critical Appraisal Skills Programme (CASP) quality assessment questions: (1) was there a clear statement of the aims of the research?; (2) is a qualitative methodology appropriate?; (3) was the research design appropriate to address the aims of the research?; (4) was the recruitment strategy appropriate to the aims of the research?; (5) was the data collected in a way that addressed the research issue?; (6) has the relationship between researcher and participants been adequately considered?; (7) have ethical issues been taken into consideration?; (8) was the data analysis sufficiently rigorous?; (9) is there a clear statement of findings?; (10) value of study and relevance to review objectives. $\checkmark=$ yes, $u=$ unsure, $p=$ partial, blank=no.

†Comments only made for those ranked moderate or low. GP, general practitioner.

moderate in value, this was due to methodological issues, and, for $8 / 13(62 \%)$ studies this was because the focus of the paper was less relevant to our research question.

Fifteen individual subthemes were identified which mapped to the seven domains of the TFA. Key findings relating to ethicality related to conflict between bisphosphonates and participants' values and were usually discussed as part of sense making. For this reason, issues relating to 'ethicality' were considered as part of 'intervention coherence', leaving six main themes, as shown schematically in figure 2. Although it was possible to distinguish between two temporal perspectives, related to anticipated and experienced acceptability within most domains (with the exception of self-efficacy) the majority of anticipated acceptability findings related to intervention coherence.

The findings of the review are discussed later with GRADE-CERQual ratings of confidence in table 3 and illustrative key findings for each theme/subtheme shown in online supplemental file 2. Subthemes are identified in the text in italics.

\section{Intervention coherence (high confidence)}

Both before starting, and during treatment, patients considered the perceived need or necessity for bisphosphonates based on their views of osteoporosis, including its seriousness and controllability, symptoms and their perception of their own health. Perceived need was weighed up against concerns about medication, including suspicion of drugs in general and specific concerns about bisphosphonate safety, by both patients and HCPs. HCPs sometimes used principles of ethicality to support perceptions of low necessity and their reluctance to prescribe. The decision process of balancing necessity against concerns, was influenced by the doctor-patient relationship and wider societal influences including friends, family and the general media. This process influenced whether HCPs reported recommending bisphosphonates. For patients, the decision process could be explicit or tacit, was revisited over time and influenced both whether they initiated treatment and subsequently adhered.

\section{Perceived effectiveness (high confidence)}

Both patients and HCPs expressed doubt or uncertainty about the mechanism of effectiveness of bisphosphonates and expressed a range of treatment expectations including strengthening bone-improving bone density, preventing worsening of osteoporosis-maintaining bone density and/or total fracture prevention. Patients wanted proof or evidence of effectiveness through more structured monitoring and follow-up, and were disincentivised to continue treatment in the absence of evidence of perceived effectiveness.

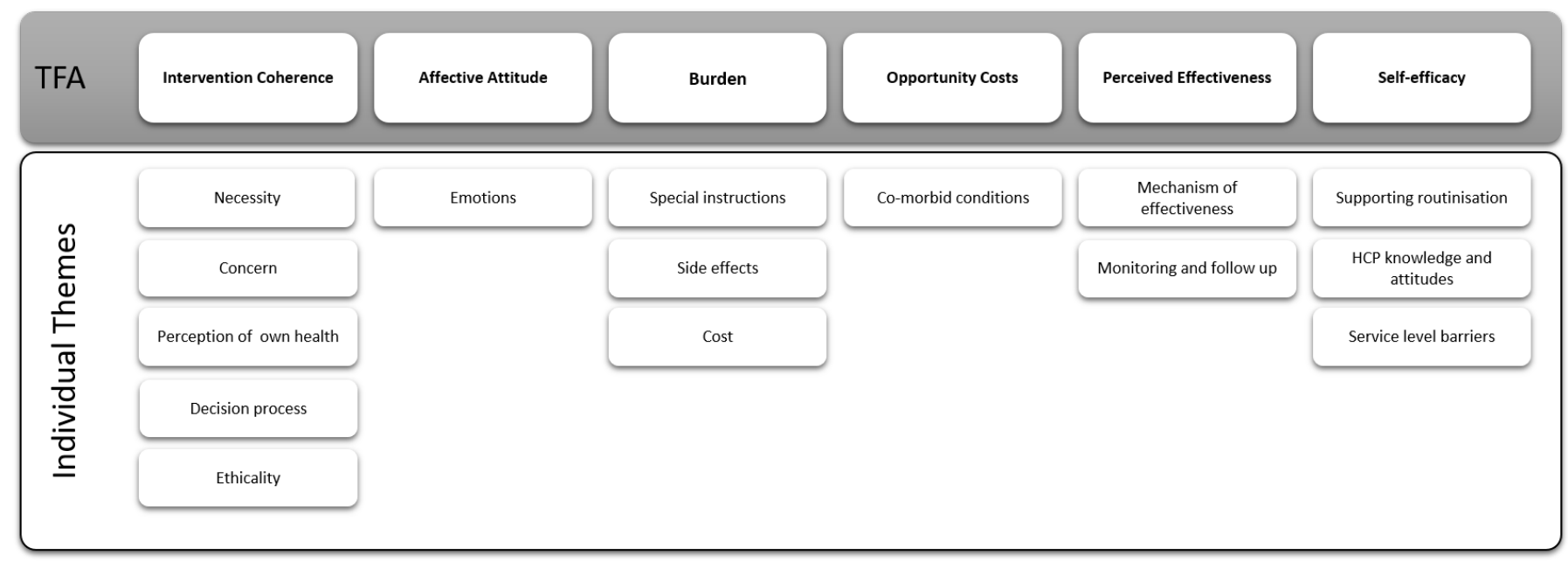

Figure 2 Identified themes and subthemes mapped to the theoretical framework of acceptability (TFA). HCP, healthcare professional. 


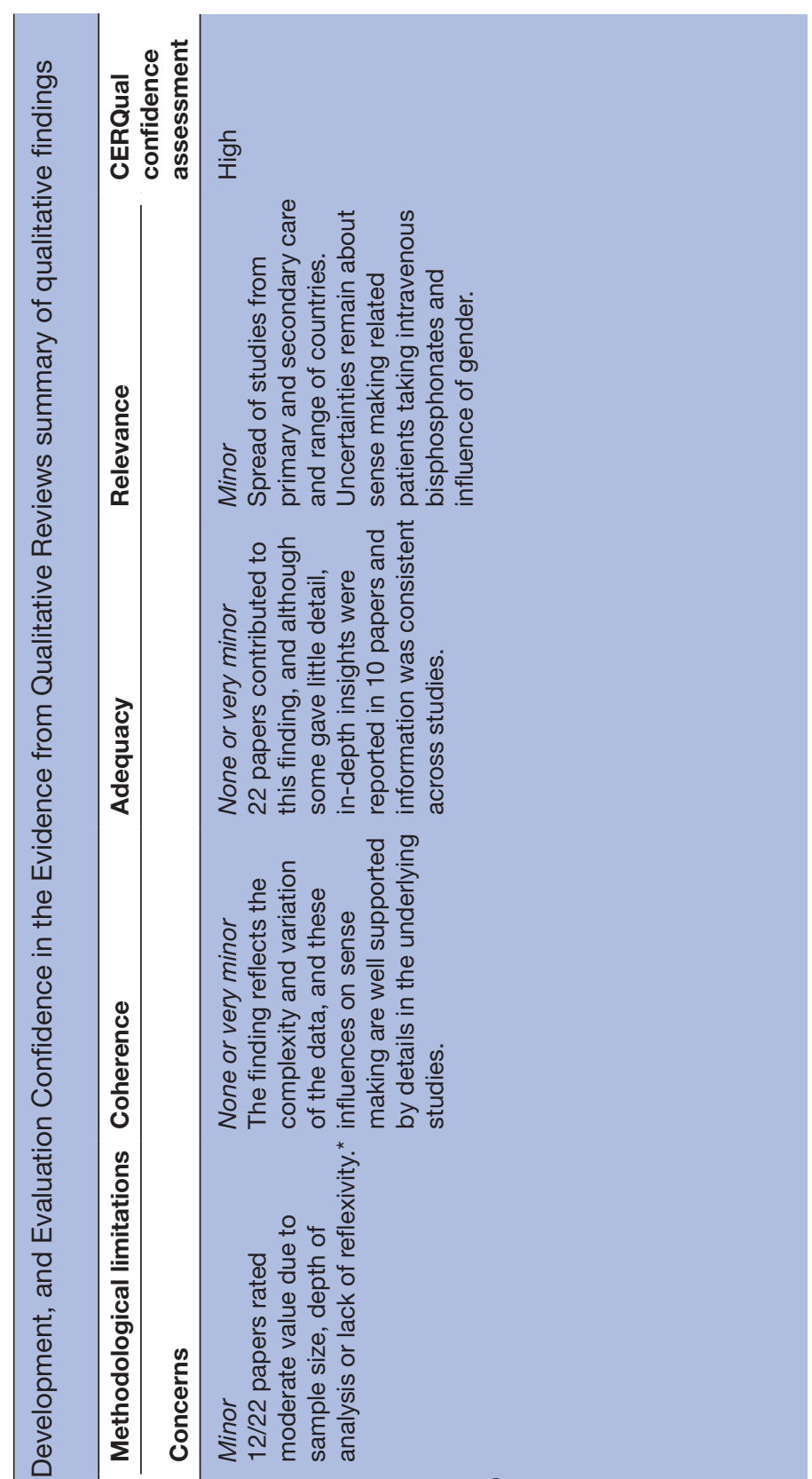

도ํ

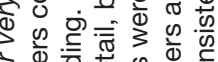

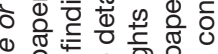

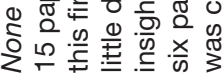

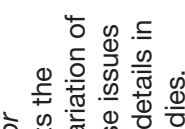

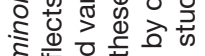
ह बे

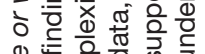
䊉

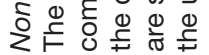

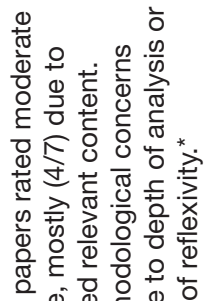

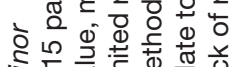

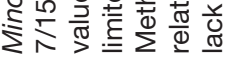

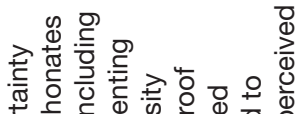

की

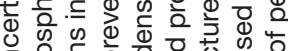

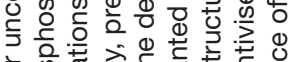
t。

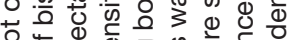

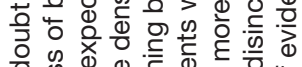
응 क

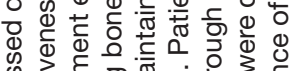

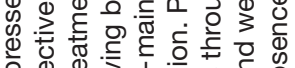

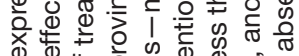

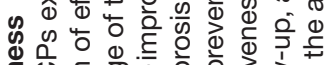

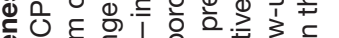

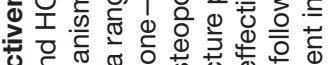

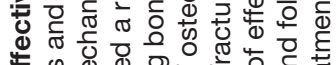

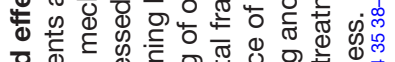

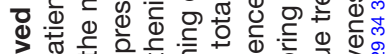

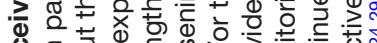

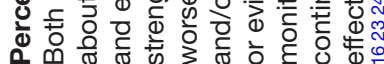




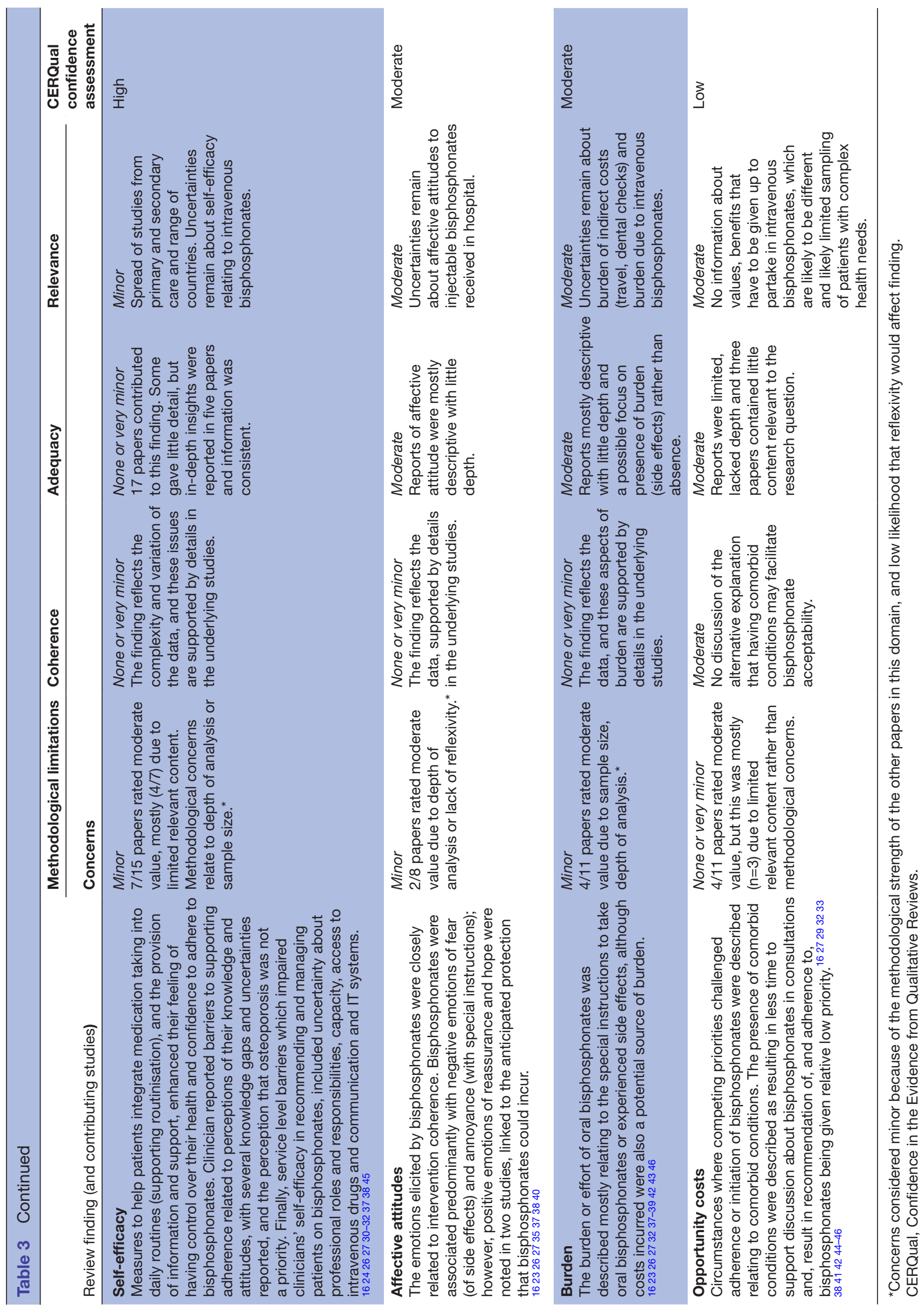




\section{Self-efficacy (high confidence)}

Measures to help patients integrate medication taking into daily routines (supporting routinisation), and the provision of information and support, enhanced their feeling of having control over their health and confidence to adhere to bisphosphonates. Clinician reported barriers to supporting adherence related to perceptions of their knowledge and attitudes, with several knowledge gaps and uncertainties reported, and the perception that osteoporosis was not a priority. Finally, service level barriers which impaired clinicians' self-efficacy in recommending and managing patients on bisphosphonates, included uncertainty about professional roles and responsibilities, capacity, access to intravenous drugs and communication and IT systems.

\section{Affective attitudes (moderate confidence)}

The emotions elicited by bisphosphonates were closely related to intervention coherence. Bisphosphonates were associated predominantly with negative emotions of fear (of side effects) and annoyance (with special instructions); however, positive emotions of reassurance and hope were noted in two studies, linked to the anticipated protection that bisphosphonates could incur.

\section{Burden (moderate confidence)}

The burden or effort of oral bisphosphonates was described mostly relating to the special instructions to take oral bisphosphonates or experienced side effects, although costs incurred were also a potential source of burden. Only one study included the experience of a patient on an intravenous bisphosphonate, this patient described low treatment burden as she only had to go once a year, and felt no side effects. ${ }^{31}$

\section{Opportunity costs (low confidence)}

There were few descriptions of 'benefits, profits, or values' being given up to take bisphosphonates. However, circumstances where competing priorities challenged adherence or initiation of bisphosphonates were described relating to comorbid conditions. The presence of comorbid conditions was described as resulting in less time to support discussion about bisphosphonates in consultations and, result in recommendation of, and adherence to, bisphosphonates being given relative low priority.

\section{DISCUSSION}

This systematic review has used the lens of acceptability to understand perceptions of bisphosphonates and the problem of poor adherence. We have identified, with high confidence, how patients and HCPs make sense (coherence) of bisphosphonates by balancing perceptions of need against concerns, how uncertainty prevails about perceived effectiveness of bisphosphonates and how a number of individual and service factors have potential to increase self-efficacy in recommending and adhering to bisphosphonates. We identified with moderate confidence, that bisphosphonate taking induces fear, but has the potential to engender reassurance, and that both the side effects and special instructions for taking oral bisphosphonates can be a source of treatment burden. Finally, we identified with low confidence that multimorbidity plays a role in people's perception of bisphosphonate acceptability.

To our knowledge, this is the first use of the TFA, originally developed to evaluate acceptability of complex interventions, to evaluate the acceptability of medication. We explored the utility of the TFA from two perspectives, as an explanatory model for both patient and clinician acceptability and engagement. The TFA was useful for understanding and combining patient and clinician viewpoints; however, there was considerable overlap between domains; perceived efficacy, affective attitudes and selfefficacy beliefs are all likely to impinge on sense-making, or intervention coherence. The TFA alone does not provide a comprehensive framework for understanding patient acceptability or engagement with medicines, and of course it was not intended to do so. The sensemaking aspect of the framework appeared pivotal, and the explanatory value of the framework was enhanced by the incorporation of the NCF to operationalise key engagement related beliefs. In the context of bisphosphonates, concern and associated fears predominate among patients, and perceived need may be underestimated if the consequences of osteoporosis and fragility fractures are not explained. In our findings, sense making was dynamic. Patients re-evaluated perceptions of bisphosphonates over time, expressing uncertainty relating to what represents successful treatment and citing perceived lack of effectiveness being cited as reason to discontinue. This is likely to be a particular problem for bisphosphonates, as opposed to other drugs commonly taken for prevention such as statins and antihypertensive, where measures of feedback and effectiveness are more readily available.

The UK National Institute for Health and Care Excellence (NICE) guidelines for medicines adherence emphasises the need to take into account perceptions (eg, necessity beliefs and concerns) and practicalities (eg, capability and resources) that will affect individuals' motivation and ability to start and continue with treatment. ${ }^{47}$ However, interventions designed to improve bisphosphonate adherence are often designed to 'educate' or persuade the patient of importance and are often not targeted to eliciting or addressing health beliefs, or informed by underpinning mechanisms of change. ${ }^{3}$ There is therefore a need to ensure that any further design of interventions-to promote bisphosphonate adherence-draws on more comprehensive theoretical models of patient engagement with health conditions and medicines such as the Extended Common Sense Model. ${ }^{48}$ This model situates individual's perceptions about drugs, and practical issues related to capability, in the context of illness and treatment representations.

Specifically, our findings suggest a need for clinicians to support patients to understand the need for treatment, to 
allay concerns where possible and to define what constitutes successful bisphosphonate treatment. Furthermore, clinicians need to support patients evaluate the advantages and disadvantages over time, given the dynamic nature of these decision processes. ${ }^{48}$

It is clear from our findings that clinicians also have necessity-concern dilemmas relating to bisphosphonates. A number of studies reported clinicians themselves perceiving low patient need, high concerns and perceptions treatment was not practical. This is perhaps in contrast with a previous quantitative study in asthma which demonstrated that clinicians held stronger positive beliefs about medicines than patients. ${ }^{49}$ It is unclear to what extent the perceptions in our findings were generalisations or applied in specific circumstances, or to what extent these views were negotiated on an individual basis in discussion with patients. Problems may arise in the consultation if clinicians assume patients share their views and then may be less likely to explore patient perceptions of need or concerns. Furthermore, the limitations of interviewing HCPs are well documented; the accounts presented in an interview may not represent clinician underlying beliefs or behaviours meaning that observational methods may be more appropriate to fully understand clinical decisional making. ${ }^{50}$ Given the clinician has a pivotal role in sense making, interventions are also likely needed to address clinician knowledge, attitudes and beliefs. By including the views of clinicians and managers we have also identified a range of service level barriers to promoting bisphosphonate adherence relating to lack of clarity about professional roles, both across primary and secondary care, and within primary care, use of IT systems and access to intravenous treatments.

A strength of this review is the comprehensive search, use of underpinning theoretical framework, the inclusion of clinician views in addition to patients, and the use of the GRADE-CERQual to give confidence in our findings which has facilitated a clear identification of where further research is needed. Areas where we have identified moderate or low confidence are in need of further research and specifically relate to the influence of multimorbidity on sense making, burden and self-efficacy in bisphosphonate users, the extent to which intravenous bisphosphonates may overcome issues related to treatment burden and self-efficacy, and the impact of bisphosphonates on affective attitudes and emotions. Furthermore, we have identified gaps in our understanding of how clinicians make decisions in practice, and how views of bisphosphonates may be influenced by gender. Given that many osteoporosis drugs have a different evidence base and licensing arrangements in men this is an area in need of further study.

The main limitation of this study relates to the lack of clarity in many of the included studies in the results sections about which osteoporosis treatments or bisphosphonates were being referred to, meaning that in some cases we may have over-interpreted findings relating to bisphosphonates that were about other osteoporosis drugs. However, all of our review findings were identified from comparison of data from several studies, and as bisphosphonates represent the mainstay of osteoporosis treatment, we consider that over-interpretation is unlikely. As there was frequently little detail about medication participants were taking or referring to, it is also possible that we have missed relevant studies. The views of men were under-represented; although 8/18 studies included men, men represented less than $20 \%$ of the total patient population in the included studies. It is important for future studies to include males and specific populations such as those with glucocorticoid-induced osteoporosis who are likely to have different experiences and needs. ${ }^{51}$ Only two studies reported the views of managers but unfortunately neither of these studies distinguished professional roles in the presentation of results, so a further need exists to explore perceptions of this group, and perceptions of payors and academics. Finally, although the population from which each study sampled was reasonably well described, it was not always possible to appreciate if the setting was primary or secondary care; the majority of studies appeared to recruit from primary care which may explain the lack of findings related to intravenous bisphosphonates and limit the transferability of our findings to non-primary care settings.

\section{CONCLUSIONS}

In summary, using the lens of acceptability, we have identified the factors that influence how patients and clinicians make sense of bisphosphonates, described the experience of bisphosphonate taking in terms of burden and factors that both facilitate and hinder confidence in taking, and prescribing and monitoring bisphosphonates. Our findings demonstrate the need for a theoretically informed, whole-system approach' to enable clinicians and patients to get the best from bisphosphonate treatment. Patients need comprehensive support that takes account of the perceptions (eg, treatment necessity beliefs and concerns) and practicalities (eg, capability and resources) that influence their motivation and ability to start and continue with treatment. Clinicians need to moderate patient expectations and clarify what constitutes bisphosphonate treatment success. Finally, further research is needed to explore perspectives of managers, patients receiving intravenous bisphosphonates, men receiving bisphosphonates and the use of bisphosphonates in the context of multimorbidity.

\section{Author affiliations \\ ${ }^{1}$ School of Medicine, Keele University, Keele, UK \\ ${ }^{2}$ Haywood Academic Rheumatology Centre, Haywood Hospital, Stoke-on-Trent, UK \\ ${ }^{3}$ Centre for Health Innovation, Leadership and Learning, University of Nottingham, Nottingham, UK \\ ${ }^{4}$ Operations and Clinical Practice, Royal Osteoporosis Society, Bath, UK \\ ${ }^{5}$ Faculty of Medicine, University of Malaya, Kuala Lumpur, Malaysia \\ ${ }^{6}$ Centre for Endocrinology Diabetes and Metabolism, University of Birmingham, \\ Birmingham, UK \\ ${ }^{7}$ Faculty of Medicine \& Health Sciences, University of Nottingham, Nottingham, UK}


${ }^{8}$ Division of Epidemiology and Public Health, University of Nottingham, Nottingham, UK

${ }^{9}$ School of Pharmacy, University College London, London, UK

${ }^{10}$ Department of Geriatric Medicine, Nottingham University Hospitals NHS Trust, Nottingham, UK

Twitter Fay Crawford-Manning @f_m_manning and Elizabeth Cottrell @cottrell_ lizzie

Contributors Conceptualisation: ZP, SB, EC, AD, TO, NG, JL-B, TL, OS. Protocol: ZP, EC, NC, SB, JL-B, TL, TO, OS, NG, AD. Search implementation: ZP, EC, NC, JW, FC-M. Data extraction and quality: ZP, EC, NC, JW, FC-M, SB. Synthesis: ZB, SB, EC, FC-M, RH, CJ. Writing- original draft: ZP, FC-M. Writing-review and editing: ZP, SB, AD, TO, NG, JL-B, CJ, TL, OS, FC-M, JW, NC, NH, EC.

Funding This study is funded by the National Institute for Health Research (NIHR), [HTA NIHR127550]. ZP is funded by the NIHR, Clinician Scientist Award (CS-201818-ST2-010)/NIHR Academy. CJ is part funded by the NIHR Applied Research Collaboration West Midlands. The views expressed are those of the author(s) and not necessarily those of the National Health Service, the NIHR, or the Department of Health and Social Care.

Competing interests None declared.

Patient consent for publication Not required.

Provenance and peer review Not commissioned; externally peer reviewed.

Data availability statement All data relevant to the study are included in the article or uploaded as supplementary information.

Supplemental material This content has been supplied by the author(s). It has not been vetted by BMJ Publishing Group Limited (BMJ) and may not have been peer-reviewed. Any opinions or recommendations discussed are solely those of the author(s) and are not endorsed by BMJ. BMJ disclaims all liability and responsibility arising from any reliance placed on the content. Where the content includes any translated material, BMJ does not warrant the accuracy and reliability of the translations (including but not limited to local regulations, clinical guidelines, terminology, drug names and drug dosages), and is not responsible for any error and/or omissions arising from translation and adaptation or otherwise.

Open access This is an open access article distributed in accordance with the Creative Commons Attribution 4.0 Unported (CC BY 4.0) license, which permits others to copy, redistribute, remix, transform and build upon this work for any purpose, provided the original work is properly cited, a link to the licence is given, and indication of whether changes were made. See: https://creativecommons.org/ licenses/by/4.0/.

\section{ORCID iDs}

Fay Crawford-Manning http://orcid.org/0000-0002-9768-1695

Elizabeth Cottrell http://orcid.org/0000-0002-5757-1854

\section{REFERENCES}

1 Cooper C, Campion G, Melton LJ. Hip fractures in the elderly: a world-wide projection. Osteoporos Int 1992;2:285-9.

2 National Osteoporosis Society. Nhs RightCare scenario: the variation between sub-optimal and optimal pathways, 2017: 1-16.

3 Hiligsmann M, Cornelissen D, Vrijens B, et al. Determinants, consequences and potential solutions to poor adherence to antiosteoporosis treatment: results of an expert group meeting organized by the European Society for clinical and economic aspects of osteoporosis, osteoarthritis and musculoskeletal diseases (ESCEO) and the International osteoporosis Foundation (IOF). Osteoporos Int 2019;30:2155-65.

4 Khosla S, Shane E. A crisis in the treatment of osteoporosis. J Bone Miner Res 2016;31:1485-7.

5 May C, Montori VM, Mair FS. We need minimally disruptive medicine. BMJ 2009;339:b2803.

6 National Institute for Health Care Excellence. Bisphosphonates for treating osteoporosis, Technology appraisal guidance (TA46) NICE Guidance, 2017.

7 Jha S, Wang Z, Laucis N, et al. Trends in media reports, oral bisphosphonate prescriptions, and hip fractures 1996-2012: an ecological analysis. J Bone Miner Res 2015;30:2179-87.

8 Recknor C, Czerwinski E, Bone HG, et al. Denosumab compared with ibandronate in postmenopausal women previously treated with bisphosphonate therapy: a randomized open-label trial. Obstet Gynecol 2013;121:1291-9.

9 Akarırmak Ülkü, Koçyiğit H, Eskiyurt N, et al. Influence of patient training on persistence, compliance, and tolerability of different dosing frequency regimens of bisphosphonate therapy: an observational study in Turkish patients with postmenopausal osteoporosis. Acta Orthop Traumatol Turc 2016;50:415-23.

10 Kishimoto $\mathrm{H}$, Maehara M. Compliance and persistence with daily, Weekly, and monthly bisphosphonates for osteoporosis in Japan: analysis of data from the Clsa. Arch Osteoporos 2015;10:27.

11 Durden E, Pinto L, Lopez-Gonzalez L, et al. Two-Year persistence and compliance with osteoporosis therapies among postmenopausal women in a commercially insured population in the United States. Arch Osteoporos 2017;12:22.

12 Saini SD, Schoenfeld P, Kaulback K, et al. Effect of medication dosing frequency on adherence in chronic diseases. Am J Manag Care 2009;15:22-33.

13 Hiligsmann M, Bours SPG, Boonen A. A review of patient preferences for osteoporosis drug treatment. Curr Rheumatol Rep 2015;17:61.

14 de Bekker-Grob EW, Essink-Bot ML, Meerding WJ, et al. Patients' preferences for osteoporosis drug treatment: a discrete choice experiment. Osteoporos Int 2008;19:1029-37.

15 Alten R, Krüger K, Rellecke J, et al. Examining patient preferences in the treatment of rheumatoid arthritis using a discrete-choice approach. Patient Prefer Adherence 2016;10:2217-28.

16 Feldstein AC, Schneider J, Smith DH, et al. Harnessing stakeholder perspectives to improve the care of osteoporosis after a fracture. Osteoporos Int 2008;19:1527-40.

17 Bliuc D, Eisman JA, Center JR. A randomized study of two different information-based interventions on the management of osteoporosis in minimal and moderate trauma fractures. Osteoporos Int 2006;17:1309-17.

18 Sekhon M, Cartwright M, Francis JJ. Acceptability of healthcare interventions: an overview of reviews and development of a theoretical framework. BMC Health Serv Res 2017;17:88.

19 DeJean D, Giacomini M, Simeonov D, et al. Finding qualitative research evidence for health technology assessment. Qual Health Res 2016;26:1307-17.

20 Carroll C, Booth A, Cooper K. A worked example of "best fit" framework synthesis: a systematic review of views concerning the taking of some potential chemopreventive agents. BMC Med Res Methodol 2011;11:29.

21 Horne R, Chapman SCE, Parham R, et al. Understanding patients' adherence-related beliefs about medicines prescribed for longterm conditions: a meta-analytic review of the Necessity-Concerns framework. PLoS One 2013;8:e80633.

22 Lewin S, Booth A, Glenton C, et al. Applying GRADE-CERQual to qualitative evidence synthesis findings: introduction to the series. Implement Sci 2018;13:2.

23 Besser SJ, Anderson JE, Weinman J. How do osteoporosis patients perceive their illness and treatment? implications for clinical practice. Arch Osteoporos 2012;7:115-24.

24 Sale JEM, Hawker G, Cameron C, et al. Perceived messages about bone health after a fracture are not consistent across healthcare providers. Rheumatol Int 2015;35:97-103.

25 Sale JEM, Beaton DE, Sujic R, et al. 'If it was osteoporosis, I would have really hurt myself.' ambiguity about osteoporosis and osteoporosis care despite a screening programme to educate fragility fracture patients. J Eval Clin Pract 2010;16:590-6.

26 Weston JM, Norris EV, Clark EM. The invisible disease: making sense of an osteoporosis diagnosis in older age. Qual Health Res 2011;21:192-704.

27 Hansen CA, Abrahamsen B, Konradsen H, et al. Women's lived experiences of learning to live with osteoporosis: a longitudinal qualitative study. BMC Womens Health 2017;17:17.

28 Otmar R, Reventlow SD, Nicholson GC, et al. General medical practitioners' knowledge and beliefs about osteoporosis and its investigation and management. Arch Osteoporos 2012;7:107-14.

29 Jaglal SB, Carroll J, Hawker G, et al. How are family physicians managing osteoporosis? qualitative study of their experiences and educational needs. Can Fam Physician 2003;49:462-8.

30 Ailabouni NJ, Nishtala PS, Mangin D, et al. General practitioners insight into deprescribing for the multimorbid older individual: a qualitative study. Int J Clin Pract 2016;70:261-76.

31 Sippli K, Rieger MA, Huettig F. Gps' and dentists' experiences and expectations of interprofessional collaboration: findings from a qualitative study in Germany. BMC Health Serv Res 2017;17:179.

32 Merle B, Haesebaert J, Bedouet A, et al. Osteoporosis prevention: where are the barriers to improvement in French general practitioners? A qualitative study. PLoS One 2019;14:e0219681. 
33 Merle B, Dupraz C, Haesebaert J, et al. Osteoporosis prevention: where are the barriers to improvement in a French general population? A qualitative study. Osteoporos Int 2019;30:177-85.

34 Drew S, Judge A, Cooper C, et al. Secondary prevention of fractures after hip fracture: a qualitative study of effective service delivery. Osteoporos Int 2016;27:1719-27.

35 Alami S, Hervouet L, Poiraudeau S, et al. Barriers to effective postmenopausal osteoporosis treatment: A qualitative study of patients' and practitioners' views. PLoS One 2016;11:e0158365.

36 Guzman-Clark JRS, Fang MA, Sehl ME, et al. Barriers in the management of glucocorticoid-induced osteoporosis. Arthritis Rheum 2007:57:140-6.

37 Lau E, Papaioannou A, Dolovich L, et al. Patients' adherence to osteoporosis therapy: exploring the perceptions of postmenopausal women. Can Fam Physician 2008;54:394-402.

38 Salter C, McDaid L, Bhattacharya D, et al. Abandoned acid? understanding adherence to bisphosphonate medications for the prevention of osteoporosis among older women: a qualitative longitudinal study. PLoS One 2014;9:e83552.

39 Iversen MD, Vora RR, Servi A, et al. Factors affecting adherence to osteoporosis medications: a focus group approach examining viewpoints of patients and providers. J Geriatr Phys Ther 2011;34:72.

40 Mazor KM, Velten S, Andrade SE, et al. Older women's views about prescription osteoporosis medication: a cross-sectional, qualitative study. Drugs Aging 2010;27:999-1008.

41 Wozniak LA, Johnson JA, McAlister FA, et al. Understanding fragility fracture patients' decision-making process regarding bisphosphonate treatment. Osteoporos Int 2017;28:219-29.

42 Swart KMA, van Vilsteren M, van Hout W, et al. Factors related to intentional non-initiation of bisphosphonate treatment in patients with a high fracture risk in primary care: a qualitative study. BMC Fam Pract 2018;19:141.
43 Scoville EA, Ponce de Leon Lovaton P, Shah ND, et al. Why do women reject bisphosphonates for osteoporosis? A videographic study. PLOS One 2011;6:e18468.

44 Sale JEM, Gignac MA, Hawker G, et al. Decision to take osteoporosis medication in patients who have had a fracture and are 'high' risk for future fracture: a qualitative study. BMC Musculoskelet Disord 2011;12:92.

45 Sturrock A, Preshaw PM, Hayes C, et al. Attitudes and perceptions of GPs and community pharmacists towards their role in the prevention of bisphosphonate-related osteonecrosis of the jaw: a qualitative study in the North East of England. BMJ Open 2017;7:e016047.

46 Sturrock A, Preshaw PM, Hayes C, et al. Perceptions and attitudes of patients towards medication-related osteonecrosis of the jaw (MRONJ): a qualitative study in England. BMJ Open 2019;9:e024376.

47 Nunes V, Neilson J, O'flynn N, et al. Medicines adherence: involving patients in decisions about prescribed medicines and supporting adherence (CG76. NICE Guidance, 2009.

48 Horne R, Cooper V, Wileman V, et al. Supporting adherence to medicines for long-term conditions: a perceptions and practicalities approach based on an extended Common-Sense model. European Psychologist 2019;24:82-96.

49 Driesenaar JA, De Smet PAGM, van Hulten R, et al. Beliefs about inhaled corticosteroids: comparison of community pharmacists, pharmacy technicians and patients with asthma. J Asthma 2016;53:1051-8.

50 Checkland K, Harrison S, Marshall M. Is the metaphor of 'barriers to change' useful in understanding implementation? Evidence from general medical practice. J Health Serv Res Policy 2007;12:95-100.

51 Beauvais C, Poivret D, Lespessailles E, et al. Understanding patients perspectives and educational needs by type of osteoporosis in men and women and people with Glucocorticosteroid-Induced osteoporosis: a qualitative study to improve disease management. Calcif Tissue Int 2019;105:589-608. 